\title{
Gravity talks: observing the universe with gravitational waves
}

\author{
Bernard F Schutz
}

\begin{abstract}
When the current upgrade of the large ground-based gravitational wave detectors LIGO and VIRGO is completed, the new science of gravitational wave astronomy will begin. In this overview I review the current status of the detector projects on the ground and in space (LISA), the kinds of signals and sources they expect to observe, and the science returns that are anticipated.
\end{abstract}

\section{Introduction}

The effort to detect gravitational waves has been one of the most remarkable examples of sustained technology development in the history of physics and astronomy. Like most kinds of instrumentation, gravitational wave detectors have been developed through many steps, each one bringing a significant improvement in performance. Unlike all other kinds of instrumentation that I am aware of, improvements in the sensitivity of gravitational wave detectors have not yet - after over 50 years of improvements - led to a single detection. In all other areas of physics and astronomy, early instruments have made at least some measurements or detections of interest, and these have stimulated and justified the next improvements in performance. This cycle of observation/improvement/observation/improvement is clearly evident in the histories of optical, radio, X-ray, and cosmic-ray astronomy during the last 50 years. For gravitational wave detectors, evolving over the same period of time, the cycle was simply improvement/improvement/improvement. The effort has been sustained, not by the success of serendipitous observation, but by the dedication of those working in the field and by the deep conviction (shared, of course, by the science-funding bodies) not only that gravitational waves of a certain amplitude

Bernard F Schutz

Max Planck Institute for Gravitational Physics (Albert Einstein Institute), Potsdam, Germany, email: bernard.schutz@aei.mpg.de 
are certainly passing through the Earth regularly, but that detecting these waves will dramatically change our view of the universe.

The 50-year effort has not been without other rewards for those involved. On the experiment side, many fascinating new technologies have been developed to allow us to make the most sensitive measurements of distance changes ever accomplished. Detector sensitivity after the current upgrades will be something like $10^{7}$ times better than the original instruments built in the 1960s [1]. This maps directly into range: our detectors will be able to detect signals from sources $10^{7}$ times further away than the early detectors could. On the theory side, the possibility of observing gravitational waves has stimulated many important developments, not least the ability to solve Einstein's equations on computers and to simulate systems that cannot be studied analytically. But the big goal of direct detections has not yet been reached.

This will not be the case for much longer. The large ground-based detectors LIGO, VIRGO, and GEO600 have already taken data with such high sensitivity that detections could have happened if we had been a bit lucky with how close the nearest event would happen; that no detections were registered was not a surprise, even if a slight disappointment. But the next phase of data-taking with LIGO and VIRGO will be different: if the instruments now being upgraded perform to specification, and if the astrophysical event rate is close to the estimates made by the collaboration [2], then the first detection is likely by around 2017, and it is very unlikely that nothing will be registered before, say, 2020.

Unlike in the other areas of astronomy that have had the normal observation/improvement cycle, we believe we actually have a pretty good idea of what the first detected gravitational wave signals will be: they will be from binary systems in which compact objects (neutron stars and/or black holes) spiral together and merge. This expectation is based on theory - extensive studies of the motion of compact objects in fully general-relativistic binaries, and of the astrophysics of these objects - which was needed during the last 50 years in order to justify the effort being put into the technology development, but which has even more importantly become an essential part of the detection chain. The accurate predictions we now have of what the gravitational waveforms from binaries and other systems (for example, gravitational wave pulsars) should look like permit us to dig deep into instrumental noise and detect with confidence signals that might otherwise have to wait for yet another cycle of instrument improvement. Good data analysis based on theoretical waveform predictions probably improves the sensitivity (equivalently, the distance reach) of our detectors by factors of 10-30 for binaries, and up to several thousand for pulsars.

Theoretical studies have led also to the development during the last 10 years of a new paradigm for the kind of observing that will be done by gravitational wave detectors. We now think of it as "listening" to the universe, rather than "watching" it, as one does with normal telescopes. Our detectors are not pointed, but rather omni-directional, like microphones. The data stream is one-dimensional, like an audio stream. The waves are detected coherently, like audio waves, rather than just bolometrically, like photons; in fact, the phase evolution of the waves contains more information about the sources than the amplitude does. All these analogies help to 
understand how we will extract information from our arrays of detectors. And in the same way that hearing complements vision in animals, gravitational wave observing will add a qualitatively new "sense" to our ability to record the universe around us: relativistic systems we don't know about, or are hidden, or are unable to radiate light can be discovered, located, and studied using our network of microphone-like detectors.

With first observations not far in the future, this meeting is a good time to look forward toward the coming gravitational wave astronomy: what the instruments are, what the sources are that are most likely to be detected, and what the kinds of information are that we are likely to be able to infer when we are finally able to hear the universe speaking to us through the medium of gravity.

\section{Light deflection and gravitational wave detection}

Since this meeting marks Einstein's work in Prague, it seems appropriate to draw a link between his work and the detection of gravitational waves. One of the subjects that drew Einstein's attention in Prague in 1911-12 was the deflection of light by gravity. It is perhaps amusing that our present method of detecting gravitational waves by interferometry also relies on the action of gravity on light.

Einstein's work in Prague [3] was his famous demonstration that light, on passing the Sun or another body, will be deflected. Because he relied essentially only on the equivalence principle (the curvature of time) and did not yet have a theory that included the curvature of space, he got only half of the right value; nevertheless it was an important advance in his own thinking.

In principle he could have predicted also that the propagation time of light would change due to the deflection, something we now call the Shaprio Effect. But as there was no way to measure this with light from a distant star, he would not have given the idea much thought. Nevertheless it is precisely these propagation-time changes that we use in detectors today. The gravitational wave makes a time-dependent alteration in the time it takes light to move up and back along the arms of a detector, and by interferometry we can compare this to the propagation time in the perpendicular arm. The signature of a gravitational wave is a difference in the propagation time alterations between the two arms.

\section{The global interferometer network}

The worldwide network of gravitational wave detectors, illustrated in Figure 1, consists of three large instruments (two LIGO and VIRGO), one medium-sized detector (GEO600), and two projects: KAGRA in Japan and LIGO-India. The existing detectors have successfully reached their first-stage sensitivity goals and demonstrated that they could operate reliably and produce data. The data have been extensively 


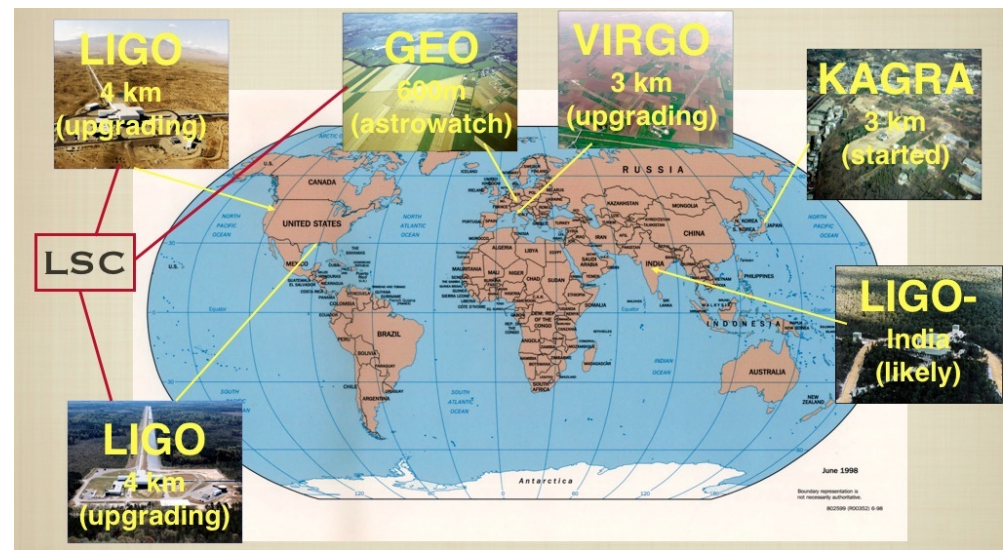

Fig. 1 The six large interferometers currently operating, upgrading, or planned.

analyzed, leading to over 70 papers reporting methods and results [4], but no gravitational wave signals were found in the data. This was not a surprise, since the first sensitivity goals were modest relative to the expected event rates, so now the three large instruments are upgrading their sensitivities by a factor of about 10, at which level it would be very surprising if no detections were made. During this upgrade, the GEO600 detector is spending about 70\% of its time in "Astrowatch" mode, meaning it is taking data just in case an interesting astronomical event occurs nearby, such as a supernova in our galactic neighborhood. The rest of the time it is also upgrading its sensitivity. The LIGO and GEO600 detectors are jointly managed and developed within the LIGO Scientific Collaboration (LSC).

GEO600 has functioned as a technology development platform as well as a detector, and a number of the important technologies that are being used in the upgrades of the larger instruments were first developed and/or tested in GEO600: high laser powers, monolithic suspensions for controlling thermal noise, signal recycling, and squeezed light. The basic installation that will turn LIGO into Advanced LIGO will be completed in 2015, and there will follow a series of commissioning periods alternating with observing runs, as the sensitivity is improved to the final goal. By 2017 it seems reasonable to expect the first detections to have occurred. Advanced VIRGO is on the same trajectory, perhaps 6 months to a year later.

KAGRA (previously LCGT) is a 3-km scale instrument with very ambitious technology: underground and cryogenic. It is now digging its tunnels in the Kamiokande mine in Japan, and should be producing data with a sensitivity comparable to that of Advanced LIGO and VIRGO before the end of the decade. The newest development is LIGO-India, in which LIGO will install a detector into a vacuum system built by India. The project has been endorsed in the US by the National Science Board, and is awaiting final funding approval at cabinet level in India.

In the longer-term future, scientists have studied so-called third-generation detectors and their technology. The most complete study was for the European Einstein 
Telescope [5, 6], which will probably bid for first funding toward the end of the current decade.

All of these instruments share data (or in the case of KAGRA and LIGO-India, plan to do so) and publish results jointly, with more than 600 authors on each paper. When one thinks about it, this degree of cooperation is unusual among physicists. Large collaborations are not uncommon, but normally there are two or more collaborations that compete with one another. In gravitational waves, all the detectors cooperate.

Such harmony is driven by the science: there are good reasons why the science benefits from pooling data. First, one could not reliably claim a detection of a short burst of gravitational radiation on the basis of the data of a single detector, because the signals are so weak that it would be easy for some un-modeled noise in a detector to masquerade as signal. Second, detectors are almost omni-directional, so to get direction information and be able to solve for the polarization of a short burst signal one needs three or more detectors, triangulating the position on the sky from time-delays among the detectors. That means that the global network itself forms an interferometer in the same sense that radio telescopes around the globe join in VLBI networks. And with four or more detectors the science gets even better: since instruments have duty cycles (for first-stage LIGO it was around 80\%), more detectors means that there is better coverage of the sky in both direction and time. And with more observed signals it is possible to extract better waveform information by averaging over the (independent) noise in the various detectors.

These advantages can be made quantitative in terms of some figures of merit for different networks, introduced in [7]. The Triple Detection Rate is a number that reflects the sensitivity of a network (as measured by the spatial volume inside its antenna pattern) and the time-coverage for an assumed duty cycle. For networks of more than three detectors it weights the product of time and volume for all threedetector sub-networks, on the grounds that one needs at least three detectors to get a reasonable amount of science from a detection. The Sky Resolution figure of merit measures the inverse of the typical area of the error box on the sky for locating an observed event. Both of these measures are given for three detector networks in Table 1 . The networks are: the originally planned LIGO-VIRGO network, with two detectors at LIGO Hanford in Washington, one at LIGO Livingston in Louisiana, and the VIRGO detector (HHLV); VIRGO with the extended LIGO network with the second Hanford detector moved to its new home in India (HILV); and the ultimate worldwide network including Japan (HIJLV). The numbers are used only to compare networks, so we do not give scalings that allow one to go from a figure of merit to a measurable quantity like the area of the error box. But the relative values are significant: HIJLV produces sky location error boxes that are a factor of 7.6 smaller in area than HHLV, for example. This and the improvement in location going from HHLV to HILV are the main motivation for building the two detectors in India and Japan. However, one also sees that the added sensitivity and time-coverage if one assumes $80 \%$ duty cycle for the new detectors will practically double the number of events that are detected with three or more detectors, comparing HHLV to HIJLV. 


\begin{tabular}{lll}
\hline Network & Triple Rate & Resolution \\
\hline HHLV & 4.86 & 0.65 \\
HILV & 5.94 & 2.96 \\
HIJLV & 8.62 & 4.60 \\
\hline
\end{tabular}

Table 1 Figures of merit for three networks (see text for explanation). Data from [7]

Anyone interested in the sociology of the international gravitational wave collaboration, about how scientists manage to cooperate and reach decisions without having a strong hierarchical organization, should read the study [8]. The author, $\mathrm{H}$ Collins, has privileged access to the inner discussions of the collaboration, which he uses for his sociological study.

\section{Data analysis}

Data analysis is an important part of any physics experiment or astronomical observation, but it plays a particularly important role in gravitational waves detection. In fact, detection with the ground-based instruments relies on three key components: the detectors' sensitivity, the accuracy of the source modeling (e.g. predicting waveforms from black hole mergers), and the sophistication of the data analysis.

The signals we expect will not be easily visible in the data streams: they will emerge only after processing the data through intelligent filters that remove noise and enhance signal. This so-called matched filtering relies, in turn, on good predicted waveforms. So, unlike in most branches of astronomy, where the modeling of the source comes after the data have been analyzed, here we need the modeling first as an input to the data analysis. And because noise can always masquerade as signal, any detection statement will be a statistical one. The LSC and VIRGO collaborations have agreed that their first claim to detection will need to have a " $5 \sigma$ " level of confidence, that there is less than one chance in three million that noise could have created the claimed signal at any time during the entire data run in which the detection was made. This will need to take into account the fact that searches are done over large parameter spaces of possible signals (different masses, spins, sky locations, etc), so that there is a large "trials factor" for all the different independent filters that have been employed, which additionally discounts the significance of a detection.

Because the noise is not an ideal Gaussian distribution, but rather has a population of random instrumental "glitches" that have to be discriminated from signals, and because the number of filters is so large that the computational demands sometimes exceed the available resources, it is generally not possible to do fully optimum signal analysis. Instead, there is a premium on clever data analysis algorithms that get closer to the theoretical optimum sensitivity. This means that if it is possible to develop an algorithm that digs deeper into the noise by a factor of 2 , then that 
algorithm effectively improves the sensitivity of the detectors to that kind of signal by a factor of 2 . Developing such algorithms is usually a lot less expensive than upgrading the hardware to achieve a sensitivity change by a factor of 2 . That is why, alongside the ongoing hardware upgrades, there is a large amount of work going into algorithm development.

But besides algorithms, accurate input from the wider research community on source and waveform models can be similarly effective in improving sensitivity. The NINJA [9] and NRAR [10] collaborations are working hard to bring numerical relativity results into the data analysis systems.

To cope with the unusually large demand for computing power of some of the searches (particularly for low-amplitude continuous signals from pulsars) the gravitational wave community has developed the Einstein@Home platform [11]. This is a screen saver that performs data analysis when a computer is otherwise idle. So many people have downloaded and participate in the search that the platform currently delivers almost 700 Tflops of performance continuously! It has also been used recently to search through radio and gamma-ray data for pulsars, with great success, discovering systems that had been missed by previous data analysis. This validates the methods and approach of the gravitational wave community in the search for long-duration signals.

Because the data analysis involves such a large amount of preparation and resource, there has been considerable discussion about the value of public data releases. Current policy by the LSC is to release small segments of data that contain confirmed signals as soon as possible. Full data releases will occur after a period of perhaps two years, once the full data analysis apparatus of the collaboration has been applied and the results understood. The collaboration is also preparing software interfaces to allow scientists not experienced in the projects to perform their own analyses. One unknown is how much support the collaboration will be funded to provide for outside analysis: much of the complication of the analysis has to do with characteristics of the detectors (un-modeled noise, etc) that may prove difficult for non-members to deal with.

\section{Observables}

The data analysis ultimately determines the values of the parameters that are the unknowns in the family of signals that are used as the template of the search, within the uncertainties of the observations. For the most commonly expected signals, from binary coalescence, the principal parameters are:

- Location on the sky. Fundamentally this comes from the differences in the arrival times of the signals at the various detectors. Actually the arrival time is not well-defined for a long-duration signal like that from a binary, so some fiducial time has to be defined. One such time would be the expected arrival time of the final coalescence signal of the system, if it consisted of two point particles. This can be predicted from the inspiral waveform. The actual merger will be more 
complicated, but the (fictional) coalescence arrival time provides a well defined time-parameter determined by fitting the theoretical waveform to the data. Three such times of arrival are enough to define two possible locations on the sky. With four detectors this ambiguity is resolved.

- Polarization. Once the direction to the source is known, two detector signals suffice to determine the two polarizations. The polarization tells us the inclination of the plane of the binary orbit to the line of sight. If the polarization changes (say through orbit precession) then only one of the two possible directions determined by three detectors will remain fixed in the sky. If there are three or more detectors and the location of the signal is known, then the two polarization signals are overdetermined, at least if general relativity is valid, and that redundancy is useful: there is a linear combination of outputs that contains no gravitational wave signal: this is called a null stream, and it can be used, for example, to veto spurious signals of instrumental origin. For $N>2$ detectors there are $N-2$ null streams. With three or more detectors it is possible to test the general-relativity model of polarization; this could reveal new gravitational fields, which are a feature of many unified field theories for quantum gravity.

- Masses and spins of the component masses. For signals that can be well modeled, the phase/frequency information tells us about the masses. For a binary, fitting a post-Newtonian waveform description to the orbit is enough to determine the individual masses and spins, provided the signal is strong enough. The parameter that is most reliably determined is the so-called chirp mass $\mathscr{M}=m_{1}^{3 / 5} m_{2}^{3 / 5} / M^{1 / 5}=\mu^{3 / 5} M$, where $m_{k}$ is the mass of the $k^{\text {th }}$ star, $M=m_{1}+m_{2}$ the total mass, and $\mu=m_{1} m_{2} / M^{2}$ the symmetric mass ratio [12].

- Distance to the source. Remarkably, binary signals from systems whose orbital frequency changes during the observation due to gravitational radiation reaction contain enough information to determine the distance to the binary system: they are standard candles, or (more appropriately) standard sirens [12]. To understand how this is possible, consider first the three observables that one can measure for a circular binary with equal masses: the frequency $f$ of the signal, its time-scale for changing $\tau_{c}=f / \dot{f}$ (called the chirp time), and the intrinsic amplitude of the wave $h$ (which is only known if the location of the signal on the sky and its polarization have also been measured). These three depend on the mass $m$ of both components, their orbital radius $a$, and the distance $d$ to the source. With three measured values, each of these three unknowns can be determined, and in fact to within factors of order unity

$$
d \sim c /\left(f^{2} \tau_{c} h\right)
$$

If the system has unequal mass components, then this formula still appiles, because only the chirp mass $\mathscr{M}$ is needed. If the system is eccentric, then there is enough information in the phase of the signal to determine the eccentricity and come back to the same formula. And if the system is at cosmological distances, then the distance measured is the luminosity distance $D_{L}$. We expect this to be a powerful added tool in astronomy, by checking the standard astronomical dis- 
tance ladder (which this method is completely independent of), by helping to identify binary sources and their host galaxies, and even by providing a local value of the Hubble constant $[12,13]$.

It is of course important to understand that not all parameters are determined equally well; in fact the errors in some of them can have strong covariances with one another. This issue is studied in detail by the search teams as they do their data analysis. The location on the sky can have strong covariances with polarization, for example. Determining the position accurately is a key for doing Multimessenger Astronomy: finding correlated signals in the gravitational wave and electromagnetic spectra. Conversely, an independently determined position (from an electromagnetic observation) will reduce the position errors and thereby improve the determination of other parameters.

\section{Gravitational waves from neutron stars and black holes}

\subsection{Neutron star binary coalescence}

The in spiral and coalescence of two neutron stars is the top candidate for the firstever detection by the ground-based network. This is because the rate of such events in the universe is fairly well understood [2] and the signal template is very accurately modeled. The best estimate is that a three-detector network of interferometers at the advanced level of sensitivity (which we expect to have from 2019 onwards) should detect something like 40 events per year. The first event could come in 2017, earlier if we are fortunate. With an enlarged network that includes LIGO-India and KAGRA, the event rate could go up to $100-150$ events per year. The maximum distance for such events will be in the range 400-600 Mpc. The enlarged network does not have a greatly increased reach. Instead, it covers the sky more isotropically, so it does not miss very many events out to this distance [7].

These events will bring a great deal of exciting science. We will have a much better sampling of the binary pulsar population, leading to mass distributions that could give clues to the prior evolution of such systems. The strongest (closest) event in any year should have a high enough signal-to-noise ratio (SNR) to provide a strong constraint on the neutron-star equation of state. These mergers are likely to produce gamma-ray bursts [14]; see Figure 2. Because such bursts are strongly beamed, they are not likely to be seen with each gravitational wave detection, but there should be a few coincidences per year. The combined gamma-ray and gravitational-wave data could also provide further insight into the physics of neutron stars [15].

By comparing the time of arrival of gravitational waves and gamma rays, one can constrain the difference in the speed of electromagnetic and gravitational waves; again, this would be a fundamental test of general relativity [16]. And by comparing the arrival times of the left- and right-handed circular polarization components of the waves (which will be possible by detailed fitting to the signal template) one can 


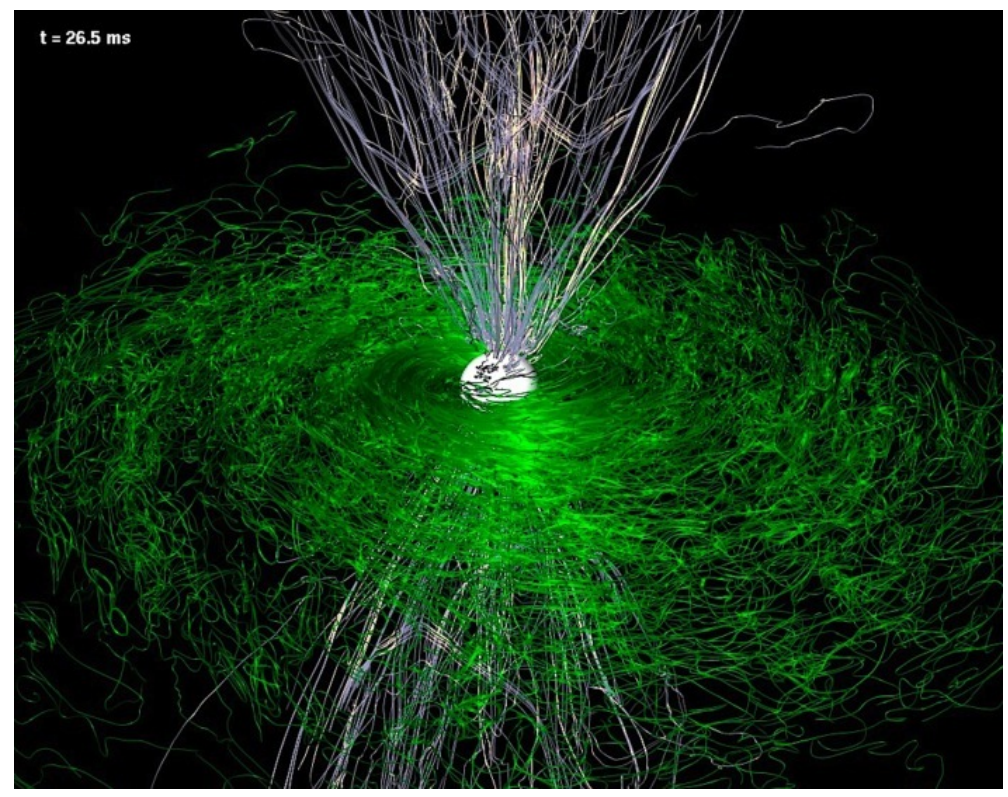

Fig. 2 A poloidal magnetic field structure that arises naturally from binary neutron-star coalescence, as computed using fully general-relativistic MHD [14].

look for birefringence in the propagation of gravitational waves, something again that is possible in modified theories of gravity [17].

The distance measures to binary systems described above, when combined with galaxy surveys and other information, could determine the local value of the Hubble constant to uncertainties less than $1 \%$ within the first 3 years [13]. When combined with accurate measurements of cosmic-microwave background measurements (as expected from the Planck mission), these coincidences might even determine the dark energy parameter $w$ to accuracies of $1 \%$ (D Holz, private communication).

\subsection{Neutron star interiors}

Neutron stars may be the most complex and extreme physical systems we know of, and because of that there is still considerable uncertainty about the physics that goes on inside them. The nuclear physics that underlies their equation of state is not accessible experimentally, and there are consequently considerable differences among the various proposed theoretical model equations. Pulsar observations tell us about strong magnetic fields, but their origin and interior distribution and strength are basically still a mystery. Therefore any information that gravitational wave observations can shed on these objects will be welcome. 
Modeling binary coalescence will certainly be one way of learning about them, as mentioned in the previous section. Another avenue will be to detect gravitational radiation from neutron star pulsation modes that may be excited by outbursts on their surfaces, which occur when newly accreted matter undergoes a thermonuclear explosion. The amount of energy that might be converted in to gravitational waves is not known, but searches in coincidence with X-ray and gamma-ray observations are planned.

\subsection{Black hole binary coalescence}

Like neutron stars, black holes are expected to be found in binary systems, some of which will decay and lead to the merger of the black holes. However, we cannot observe a population of binary black holes so we have less reliable statistics on the rates we can expect. The best estimates suggest that the observed rate of coalescence will be similar to that of neutron stars: a sparser population will be detected to greater distances due to the larger masses [2]. Within the uncertainties it is perfectly possible that the first detection will be a binary black hole merger.

These observations will enormously increase our understanding of the black hole population: masses, spins, binary mass ratios. By comparing with numericalrelativity simulations, which are now very accurate, it may be possible to test general relativity, particularly cosmic censorship, which is the hypothesis (still unproven) that a merger will always lead to a black hole and not a naked singularity. The properties of the final black hole can be inferred from its ringdown pulsation spectrum, if observed.

Black hole probably also form binaries with neutron stars, although as yet no pulsar has been detected in orbit around a black hole. Observations of these mergers with gravitational waves would be very interesting from the point of view of binary evolution theory and also to constrain the mechanisms for producing gamma-ray bursts.

\subsection{Gravitational wave pulsars}

Spinning isolated neutron stars will emit gravitational waves if they are asymmetric. The asymmetries must be small, of course, or known spinning pulsars would by now have lost their spin to the emission of gravitational waves. This consideration sets an upper limit on the expected amplitude (the spindown limit). For two pulsars, the Crab and Vela, current gravitational wave observations have constrained the amplitudes more tightly than the spindown limit [18, 19]. Advanced detectors may detect such radiation or constrain many more systems.

The search for such pulsars is very demanding computationally, and blind searches (for systems not already known as radio pulsars) are only possible with the volunteer- 
computing platform Einstein@Home [20]. In fact, the power of this system is already being used to find weak pulsar signals in radio telescope data [21], as mentioned earlier.

\section{Other gravitational wave sources}

Supernova explosions motivated the first bar gravitational wave detectors [1]; the expected $\mathrm{kHz}$ frequencies matched what bars could detect, and it seemed reasonable to think that these explosions were asymmetric enough to produce strong radiation. Recent studies, however, suggest that the amplitudes to be expected are rather small. This has to do with the difficulty that stars have in producing supernovae in the first place: the collapsed interior hangs up at large densities for a relatively long time before finally acquiring enough energy to explode. During this time, any asymmetries from the initial collapse are reduced, and the final explosion is dominated by outflow rather than oscillation, although it is possibly very turbulent (which can lead to stochastic gravitational wave emission). See, e.g. [22], for a recent study in this rapidly developing field.

Another signal that is certain to be present, but is expected to be very weak, is a stochastic gravitational wave background. There are many sources of such backgrounds, including all the binary systems in the Galaxy. At nanohertz frequencies, where pulsar timing arrays operate, this is expected to be the dominant signal and to be detectable within the next ten years [23]. But in the ground-based frequency band these signals are likely to be weaker. A big prize would be to detect a background generated by the Big Bang, but current estimates suggest it is rather weak.

\section{Detecting gravitational waves from space}

\subsection{LISA and ELISA}

So far this review has focussed on the imminent detection of gravitational waves by ground-based interferometers. But many of the people involved in the groundbased activity are also helping to develop the project to put a detector into space. The proposal has long been known as LISA, although events have recently led to a descoped version called eLISA, described below. I shall use the term LISA as a generic descriptor of a concept that is at least the long-term science goal of the field, of which eLISA will be perhaps its first realization.

Gravitational waves at frequencies below about $1 \mathrm{~Hz}$ will be very difficult or impossible to detect from the Earth because the Earth's gravity is noisy at these frequencies, and no detector could be made to respond to cosmic gravitational waves and not also to terrestrial Newtonian gravity fluctuations. Seismic density distur- 
bances, air pressure/density changes, and a host of other phenomena with timescales between 1 second and 1 hour would produce responses in detectors that are larger than those expected from cosmic sources. The only way to observe in this frequency band is to get away from the Earth, into space. The LISA proposal has been developed since 1995 within ESA, and then since 1998 jointly with NASA. Along with this proposal, ESA has been developing the LISA Pathfinder (LPF) mission, whose purpose is to demonstrate and prove the novel aspects of the LISA measurement technology that cannot be tested on the ground. LPF must be flown before the final go-ahead can be given to build a LISA mission. NASA is a minor partner in LPF.

Unfortunately, in 2011 NASA, beset with cost overruns on other missions, withdrew from its partnership with ESA on LISA, and also on two other proposed large missions. ESA then asked the three proposals to descope and enter a competition for an ESA-only large mission launch around 2021. This competition was won in mid2012 by JUICE, a mission to the moons of Jupiter. Interestingly, eLISA/NGO, the descoped gravitational wave mission, seems to have received the highest scientific rating by the ESA committees, but was nevertheless not selected, primarily because LPF has not yet launched. Since LISA Pathfinder is expected to be launched by 2015, it is not unreasonable to think that the chances of success for eLISA in the selection for the next large mission will be very good indeed. The eLISA team is therefore working hard to understand better the science capabilities of the instrument and at the same time to explore the possibility of a partnership with one or more other agencies (including NASA) that might bring in enough resources to restore some of the features of the original LISA proposal. The science document of the 2012 eLISA/NGO proposal is on the arXiv [24] and a summary appeared as a conference proceeding [25].

The LISA concept is to put a long-baseline interferometer into space, with armlengths not much shorter than the wavelengths of the gravitational waves being detected. This requires three spacecraft in an L-shaped configuration with separations of order $10^{6} \mathrm{~km}$. In the original LISA concept these spacecraft form an equilateral triangle, and laser light is used along all three arms to monitor the small changes in light travel-time along the arms produced by gravitational waves, as illustrated in Figure 3. In this way one can construct three different interferometers by using, in turn, each spacecraft as the central hub of a two-arm interferometer. These three interferometers measure different polarizations of the incoming radiation. If general relativity is correct then the output signals will be linearly dependent; checking this is a good test of the general relativity model for gravitational waves.

If the array is placed with its center on the Earth's orbit, some $20^{\circ}$ or so away from the Earth, and if the plane of the array is tilted by $60^{\circ}$ to the plane of the Earth's orbit, then when the spacecraft are given suitable initial velocities they will remain in an equilateral triangle, which rotates counter to the direction of the orbit, without any need to maintain the positions of the spacecraft: they simply follow their Newtonian orbits around the Sun. The spacecraft do need to carry thrusters, but only to resist external perturbations, such as fluctuations in solar radiation pressure. The ends of the interferometers' arms are defined by small cubes (called test masses) that fall freely in cavities inside the spacecraft (not attached to the spacecraft). The 


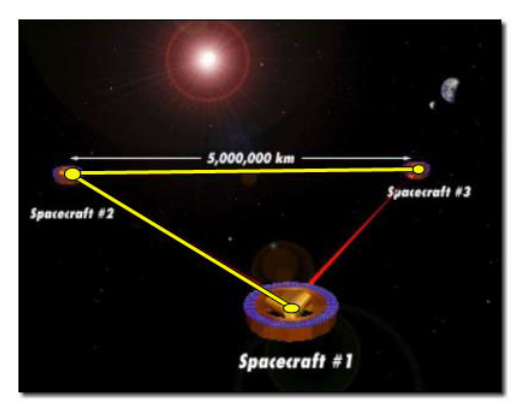

Fig. 3 A schematic illustration of the LISA concept of three equidistant spacecraft in orbit around the Sun, forming three interferometers. With an appropriately chosen orbit, the array remains an equilateral triangle even as the spacecraft orbit the Sun freely.

spacecraft effectively act as shields protecting the cubes from external perturbations. In this way the cubes follow geodesics of the gravitational field closely enough to track the disturbances produced by gravitational waves.

The LPF mission consists of a single spacecraft within which are two experiment packages like those that mark the ends of the LISA arms. Therefore, LPF contains a single LISA arm, only very short: half a meter instead of several million meters. This means that LPF can prove the measurement technology of LISA (by measuring changes $\delta L$ in the proof-mass separation to the accuracy needed by LISA) but cannot measure gravitational waves because the baseline $L$ is so short that the smallest strain that can be measured, $\delta L / L$, is far larger than any expected from astronomical systems.

The eLISA proposal was descoped to save launch weight and some component costs. It must still have three spacecraft, but only two of the arms are monitored with laser light, so there is only one interferometer signal. Although this reduces the information available from the mission, eLISA would still do spectacular science. In the following I describe this science without distinguishing too much between LISA and eLISA; this is because events in the near future might lead to further changes in the design of the mission that eventually is flown by ESA.

\subsection{LISA Science}

By virtue of its long arm lengths, any LISA-like mission will detect some events with extraordinarily high signal-to-noise ratios, as compared with our expectations for ground-based observing. Mergers of comparably sized massive black holes $\left(10^{6} M_{\odot}\right)$ can register SNR of up to 1000 in eLISA, and 5 or ten times higher in LISA. EMRI events, in which a stellar-mass black hole is captured from a nearly circular orbit by a massive black hole, can typically have SNR around 50-100. With such strong signals, it is possible to do stringent tests of gravity theory. EMRI events 
will probe whether the metric around central black holes in galaxies really is the Kerr metric. Merger events will give a detailed picture of how horizons merge (which can be compared with accurate numerical simulations) and will measure the spectrum of the ring-down radiation emitted as the final black hole settles down. From this we expect to measure the mass and spin of the hole, and thereby test cosmic censorship: is it a "clothed" Kerr metric or is it a naked singularity with $a>M$ ?

The astrophysics return will be similarly impressive and important. Any LISAlike mission will be able to detect comparable-mass black hole mergers at redshifts of 15 and beyond, if there are any that happen in the frequency band of the instrument (equivalently in the mass range $10^{4}-10^{5} M_{\odot}$ ). These would be the earliest individual astronomical systems ever detected and studied by any observing method. They would tell us much about how galaxies formed, since black holes are believed to be tracers, embedded in proto-galactic clouds, merging with one another when their host clouds merge. A three-arm LISA would have enough information to measure distances to individual events, which when combined with known cosmological parameters would give the redshifts directly. With a two-arm eLISA mission, distance measurements have large but finite errors, and when these are averaged over an ensemble of detected events it will still be possible to discriminate among different models of early galaxy and black hole evolution [25, 24].

The mergers of relatively nearby pairs of massive black holes (out to $\mathrm{z}=2$ ) are likely to provide a link between mergers and their host galaxies. With positions provided by the three-arm LISA configuration, it should be possible to identify the galaxies since their morphology should be disturbed by having experienced the gradual spiralling together of the holes over hundreds of millions of years. This will be harder with eLISA data, but with both missions there will also be an intensive search for time-linked counterparts to the merger: enhanced X-ray, optical, or radio emission compared to observations of the same area of the sky in previous epochs. Such studies should provide clues to the mechanisms of jet emission and other phenomena associated with active galaxies.

The EMRI population will for the first time give us a relatively unbiased sample of massive black holes in the centres of galaxies. This will determine the mass distribution of these black holes and also help understand the nature of the star clusters in their immediate neighbourhoods.

White dwarf binary systems in our Galaxy will actually dominate the LISA data stream at low frequencies: there are enough systems in tight orbits to provide a confusion background for LISA below $1 \mathrm{mHz}$, and a large number of these systems will be directly detectable by both LISA and eLISA. This will again provide, for the first time, an unbiased sampling of this important population, which represents the endpoint of evolution of most binary star systems. The soon-to-be-launched Gaia [26] mission will identify thousands of such systems, which can then be matched with their gravitational wave counterparts by identifying systems with the same orbital periods. Distances provided the gravitational wave measurements (for systems which change their orbital period because of the emission of gravitational waves) will then calibrate the absolute brightness of the components. 


\section{Conclusion}

The development of detectors has taken place over many decades, essentially because most of the key technologies had to be developed to the required sensitivity level within the field itself and could not be taken over from other areas of astronomy of physics. But within a very few years, ground-based detectors should be making observations with regularity, and as the network grows there could be events every couple of days. Space-based detectors will take longer to realise, again because the technology has to be developed and proved to the required sensitivity and degree of reliability demanded by space missions.

Driving these proposals is the completely new science that detectors can return, the new way of listening to the universe that provides unique information essentially orthogonal to that provided by telescopes. We will see these science returns affecting astronomy very soon, in the areas of neutron star physics, gamma-ray bursts, stellar evolution, black hole studies, and the search for gravitational wave pulsars. The science that can be delivered by a LISA-like space-based detector is recognised as having a high priority, and I am confident that we will see the launch of such a detector approved within the next few years, allowing us finally to listen to the the low-frequency whispers of our universe.

\section{References}

1. J. Weber, Gravitational radiation, Phys. Rev. Lett. 18, 498 (1967)

2. J. Abadie, K. Belczynski, others (LIGO Scientific Collaboration, V. Collaboration), Predictions for the rates of compact binary coalescences observable by ground-based gravitationalwave detectors, Classical and Quantum Gravity 27(17), 173001 (2010)

3. A. Einstein, "ber den Einfluss der Schwerkraft auf die Ausbreitung des Lichtes, Annalen der Physik 35 (1911)

4. LSC Publications. URL http://www.lsc-group.phys.uwm.edu/ppcomm/ Papers.html

5. M. Punturo, M. Abernathy, F. Acernese, et al., The third generation of gravitational wave observatories and their science reach, Classical and Quantum Gravity 27(8), 084007 (2010)

6. B. Sathyaprakash, M. Abernathy, F. Acernese, et al., Scientific objectives of Einstein Telescope, Classical and Quantum Gravity 29(12), 124013 (2012)

7. B.F. Schutz, Networks of gravitational wave detectors and three figures of merit, Classical and Quantum Gravity 28(12), 125023 (2011)

8. H. Collins, Gravity's Shadow: The Search for Gravitational Waves (University of Chicago Press, Chicago, 2004)

9. P. Ajith, M. Boyle, D.A. Brown, et al., The NINJA-2 catalog of hybrid postNewtonian/numerical-relativity waveforms for non-precessing black-hole binaries, Classical and Quantum Gravity 29(12), 124001 (2012)

10. Home Page. URL https://www.ninja-project.org/doku.php?id=nrar: home

11. Einstein@Home Project Home Page. URL http: // einstein.phys.uwm.edu/

12. B.F. Schutz, Determining the Hubble Constant from Gravitational Wave Observations, Nature 323, 310 (1986)

13. W. Del Pozzo, Inference of cosmological parameters from gravitational waves: Applications to second generation interferometers, Phys. Rev. D 86(4), 043011 (2012) 
14. L. Rezzolla, B. Giacomazzo, L. Baiotti, et al., The missing link: Merging neutron stars naturally produce jet-like structures and can power short gamma-ray bursts, Astrophys. J. Lett. 732, L6 (2011)

15. S. Nissanke, D.E. Holz, S.A. Hughes, N. Dalal, J.L. Sievers, Exploring short gamma-ray bursts as gravitational-wave standard sirens, Astrophys. J. 725, 496 (2010)

16. C.M. Will, Bounding the mass of the graviton using gravitional-wave observations of inspiralling compact binaries, Phys. Rev. D 57, 2061 (1998)

17. S. Alexander, N. Yunes, Chern-Simons modified general relativity, Physics Reports 480, 1 (2009)

18. B. Abbott, Beating the spin-down limit on gravitational wave emission from the Crab pulsar, Astrophys. J. Lett. p. in press (2008)

19. J. Abadie, B.P. Abbott, R. Abbott, et al., Beating the spin-down limit on gravitational wave emission from the Vela pulsar, Astrophys. J. 737, 93 (2011)

20. J. Aasi, J. Abadie, B.P. Abbott, et al., Einstein@ Home all-sky search for periodic gravitational waves in LIGO S5 data, ArXiv e-prints [arXiv:1207.7176 [gr-qc]] (2012)

21. B. Knispel, P. Lazarus, B. Allen, et al., Arecibo PALFA survey and Einstein@Home: Binary pulsar discovery by volunteer computing, Astrophys. J. Lett. 732, L1 (2011)

22. C.D. Ott, E. Abdikamalov, P. Moesta, et al., General-relativistic simulations of threedimensional core-collapse supernovae, ArXiv e-prints [arXiv:1210.6674 [astro-ph.HE]] (2012)

23. A. Sesana, A. Vecchio, Gravitational waves and pulsar timing: stochastic background, individual sources and parameter estimation, Class. Quant. Grav. 27, 084016 (2010)

24. P. Amaro-Seoane, S. Aoudia, S. Babak, et al., eLISA: Astrophysics and cosmology in the millihertz regime, ArXiv e-prints [arXiv:1201.3621 [astro-ph.CO]] (2012)

25. P. Amaro-Seoane, S. Aoudia, S. Babak, et al., Low-frequency gravitational-wave science with eLISA/NGO, Classical and Quantum Gravity 29(12), 124016 (2012)

26. C. Turon, K.S. O'Flaherty, M.A.C. Perryman, The three-dimensional universe with Gaia (ESA Special Publication SP-576, 2005). URL http://sci.esa.int/gaia/37100 